By:

R.E. Hebner

14th International Conference on Dielectric Liquids (ICDL), Graz, Austria, July 7-12, 2002

PR - 324

Center for Electromechanics

The University of Texas at Austin

PRC, Mail Code R7000

Austin, TX 78712

(512) $471-4496$

March 1, 2002 


\title{
Factors Contributing To Streamer Morphology
}

\author{
Robert E. Hebner \\ The University of Texas at Austin \\ Austin, TX 78712 \\ USA
}

\begin{abstract}
Recent work shows that the range of morphology of anode streamers in liquid dielectric breakdown is predicted when it is represented as stochastic growth of a branching fractal tree. This model may be analogous to the critical volume model of breakdown used in gases. Assuming there is a relationship, leads to a concept of how affecting the electron production and recombination rates in a fluid can affect streamer behavior. These concepts were tested by measuring the effect on streamer behavior due to adding a commercial antistatic additive to toluene. The results were consistent with the concepts of the model but insufficient to define the limits of the model's applicability.
\end{abstract}

\section{INTRODUCTION}

Recent work by Fowler, Devaney, and Hagedorn [1] has simulated the fast positive streamer stage of liquid breakdown as stochastic growth of a branching fractal tree. Key attributes of that model were:

1. There existed a threshold voltage for a location adjacent to an existing streamer below which no growth could occur. The threshold was determined by examining the voltages in locations adjacent to the electrode tip.

2. The probability of growth from one location to an adjacent location is weighted by a power of the voltage difference.

These attributes are mathematical assumptions. They are physically plausible but are not rooted in a model of the discharge physics. These assumptions, however, are key to developing predicted growth patterns that are consistent with experimental observations. For example, the threshold assumption provides for no possibility of growth in areas sufficiently shielded by the previous growth. The concept of such shielding builds on the early work of Kelley and Hebner [2], who showed that the electric field distribution in advance of a propagating streamer is consistent with the assumption that the streamer is a conductor. Subsequent work by Lesaint and Massala [3, 4] showed that limiting lateral streamer growth affected streamer growth in a manner that was consistent with increasing the electric field strength in the direction of propagation.

The weighting function yielded changing streamer morphology as the weighting was changed. They achieved sparse growth with a $4^{\text {th }}$-power weighting and dense growth with linear weighting. This morphology change was consistent with earlier observations of various modes of propagation of anode streamers [5].

Comparing the predictions of this modeling approach with published experimental observations provides some insight as to physical processes that may be consistent with the model assumptions. First, it is useful to consider those experimental conditions that enhance the formation of streamers like those modeled by a linear weighting of the streamer formation probability, i.e. the more dense streamers:

- Lesaint and Massala [6] studied the effects of voltage changes on positive streamer propagation in large oil gaps. At increasing levels of overvoltage, they find the streamer forms broader branching patterns.

- A number of works [7, 8, 9] have shown that the addition of aromatic additives to insulating liquids increases the branching density.

There are also experimental conditions that have been shown to suppress the bushier streamers (type 1 in the language of Hebner [5] or weighted by a linear function in the language of Fowler et al [1]). These include:

- At high voltage levels prior to streamer initiation, the branching density is decreased. McKenny and McGrath [10] have shown, using 300 ns rise-time voltage pulses, that if the voltage level is held near the level needed for streamer initiation, streamers with high branching density are produced. If, however, the voltage is raised well beyond that required for streamer onset prior to the streamer initiation, a sparse streamer is produced. 
- $\quad$ The same authors also noted [10] that, in their apparatus, more rounded needles tended to produce less highly branched streamers.

\section{COMPONENTS OF A MODEL}

These theoretical and experimental investigations suggest possible physical models that are useful to guide further work. The observations and model summarized above appear to be consistent with a process in which a free or nearly free electron is produced in a small volume near the streamer or the electrode if the streamer has not yet formed. Definitive mechanisms for this production have not yet been elucidated. Onsager [11], however, has shown, for ion-electron pairs produced by photon or collisional ionization, an external field increases the probability that the electron escapes recombination. Subsequent experiments [12] on photoionization from excited states in insulating liquids have shown the production and extraction of thermalized electrons in hydrocarbon fluids.

A model that can be postulated is one analogous to the critical volume model used in gases [13]. Conceptually, the critical volume is a volume whose boundary nearest the streamer or electrode is sufficiently far from the streamer, e.g. a few mean free paths, so an electron in that volume can produce its own streamer as it joins the initial streamer. The outer boundary is defined as the distance from the initial streamer or electrode at which an electron can gain sufficient energy from the electric field in one mean free path to produce collisional ionization. So, the critical volume is dependent on the dynamic electric field distribution.

From the definition of the critical volume, $V_{c}$, the probability, $\mathrm{dP}$, that an electron will be accelerated from the undisturbed fluid to the propagating streamer, during time dt, with sufficient energy to produce additional ionelectron pairs as it propagates is

$\mathrm{dP}=(1-\mathrm{P}) \int_{\mathrm{Vc}}(\mathrm{dn} / \mathrm{dt})_{\mathrm{e}} \mathrm{P}_{\mathrm{o}} \mathrm{dVdt}$

where (1-P) is the probability a breakdown has not already occurred in the volume element, $(\mathrm{dn} / \mathrm{dt})_{\mathrm{e}}$ is the instantaneous rate of electron production, $\mathrm{P}_{0}$ is the probability an electron does not recombine with the ion from which it detached, and $\mathrm{dV}$ is a volume element, and the integration is over the critical volume.
There is an important extension of the critical volume approach as applied to liquids suggested by the modeling of Fowler et al [1]. In gases, the critical volume is assumed to be near the electrode and, once a streamer is initiated, other processes govern its propagation. For liquids, the assumption is this process is repeated until the streamer connects the electrodes.

Assuming for the field strengths of interest the electron production is independent of the field strength, the only field strength dependence is in $\mathrm{P}_{0}$. Onsager [11] showed that probability could be expressed as a power series expansion of the field. This is conceptually consistent with the analysis by Fowler et al [1] in that it ties the probability of the existence of an appropriate electron to the field. It should be noted this model is related to, but different from, that proposed by Billar [14]. Here it is assumed electron production is a random and infrequent event largely independent of the applied field. The primary role of the field is to reduce the probability of recombination after an ion-electron pair is produced. In Billar's work, however, he assumes the fluid has "easily ionizable" molecules that are ionized by the field.

\section{EXPERIMENTAL INVESTIGATIONS}

To examine the limitations of this conceptual model, comparisons are made with measurements of the effect of using a commercial antistatic additive [15]. This experimental approach showed promise to increase the production of effective electrons, i.e. to increase $(\mathrm{dn} / \mathrm{dt})_{\mathrm{e}}$ as in (1). High-speed photographic measurements of streamer morphology were made using pure toluene and toluene with 10 parts per million by volume of ASA-3, a commercial antistatic additive for hydrocarbon fuels. The anti-static additive dissociates into positive and negative molecules that increase the low frequency electrical conductivity of the liquid. During the course of an electrical breakdown, these molecules will not move, but they are expected to increase the instantaneous rate of electron production and/or to reduce the attachment probability. The measurements were made for positive streamers in a $3 \mathrm{~mm}$ point-plane gap. The applied voltage was pulsed with rise and fall times of about $1 \mu \mathrm{s}$ and duration of about $6 \mu \mathrm{s}$. The results are summarized in Table 1. 
Table 1. Summary of streamer propagation data

\begin{tabular}{|l|l|l|}
\hline Voltage & Fluid & $\begin{array}{l}\text { Average } \\
\text { Streamer } \\
\text { Velocity }\end{array}$ \\
\hline $48 \mathrm{kV}$ & Toluene & $1.5 \times 10^{6} \mathrm{~cm} / \mathrm{s}$ \\
\hline $48 \mathrm{kV}$ & $\begin{array}{l}\text { Toluene plus } \\
\text { ASA-3 }\end{array}$ & $1.5 \times 10^{6} \mathrm{~cm} / \mathrm{s}$ \\
\hline $60 \mathrm{kV}$ & Toluene & $1.6 \times 10^{5} \mathrm{~cm} / \mathrm{s}$ \\
\hline $60 \mathrm{kV}$ & $\begin{array}{l}\text { Toluene plus } \\
\text { ASA-3 }\end{array}$ & $3.0 \times 10^{4} \mathrm{~cm} / \mathrm{s}$ \\
\hline
\end{tabular}

\section{DISCUSSION}

Qualitative inspection of the data suggests the additive converts the streamer morphology from sparse to dense as would be expected from the fractal model. The resolution of the photographs makes this observation too subject to interpretation to yield definitive results. The average velocity data, however, is sufficiently robust to make the measured differences significant. At the $48 \mathrm{kV}$ level, the standard deviations for the two data sets were less than $20 \%$. At $60 \mathrm{kV}$, the standard deviations were about $40 \%$. These large standard deviations result from the fact the growth is not uniform with time, consistent with the assumed growth pattern in the fractal model.

The primary reason the velocity is lower and the standard deviation is higher for the higher applied voltage is, at the higher voltage, the streamers stopped, or nearly stopped, growing for periods up to a few tenths of a microsecond. This effect was more pronounced in the doped than the pure toluene. Conceptually, these results are consistent with the fractal model [1] and the experiments of Lesaint and Massala [4]. Specifically, there are ample electrons available for streamer growth and more with the additive than without. At a high enough voltage, these electrons are accelerated to produce a highly branched structure that effectively smoothes and reduces the electric field in front of the streamer, producing a small critical volume further from the streamer. At that point, the streamer growth is interrupted until an electron is produced in the new critical volume.

Similarly, this conceptual approach provides an explanation of the difference between the set of results presented here and those by Lesaint and Massala [4] and the set of results presented by McKenny and McGrath [10]. With their faster pulse rise time, particularly if they had fewer effective electrons in their fluid, they could produce a very large critical volume prior to streamer initiation. A large critical volume makes it more likely a longer streamer will be produced initially. The longer streamer could produce more field enhancement, making it increasingly likely a faster streamer would develop. So, differing electron production and recombination rates coupled with different pulse shapes suggest these seemingly contradictory results can both be expected outcomes from the same model.

\section{SUMMARY}

This work explores key connections between the fractal model and the observable physical processes. A strength of the model is that it demonstrates excellent correlation with observed streamer morphologies. The challenge of the model is that the key parameter, the voltage dependence of growth of a given size in a given direction, likely depends on factors such as the electron production and recombination rates that are not easily amenable to direct experimental verification. This work that attempts to vary these rates can produce results consistent with the conceptual basis of the model.

\section{ACKNOWLEDGEMENTS}

The author is indebted to Drs. Howland Fowler, Edward Kelley, Gerald Fitzpatrick, and Eric Forster for teaching him the theoretical and experimental approaches to understanding breakdown in dielectric liquids. Particular thanks is due to Dr. Kelley, who spearheaded the development of the equipment and procedures used to acquire the streamer photographs that were analyzed to support this paper.

\section{REFERENCES}

[1] Fowler, H.A.; Devaney, J.E.; Hagedorn, J.G. "Shaping of Filamentary Streamers by the Ambient Field,” Annual Report of the 1999 Conference on Electrical Insulation and Dielectric Phenomena, IEEE Publication 99CH36319, pp. 132 -136.

[2] Kelley, E. F. and Hebner R. E., "The Electric Field Distribution Associated with Pre-breakdown Phenomena in Nitrobenzene,” J. Appl. Phys., Vol. 52, No. 1, January 1981.

[3] Lesaint, O. and Massala, G., "Transition to Fast Streamers in Mineral Oil in the Presence of Insulating Solids,” Conference Record of the 1996 International Symposium on Electrical Insulation, IEEE Publication 96CH35972, pp.737 - 740. 
[4] Lesaint, O. and Massala, G., "Positive Streamer Propagation in Large Oil Gaps - Electrical Properties of Streamers," IEEE Trans. DEI, Vol. 5, No. 3, June 1998, pp. $371-381$.

[5] Hebner, R. E., "Measurement of Electrical Breakdown in Liquids", The Liquid State and Its Electrical Properties, Kunhardt, E. E., Christophorou, L. G., Luessen, L. H., eds., Plenum Press, New York, pp. 519 - 537, 1987.

[6] Leasint, O. and Massala, G., “ Positive Streamer Propagation in Large Oil Gaps - Experimental Characterization of Propagation Modes,” IEEE Trans DEI, Vol. 5, No. 3, June 1998, pp. 360 - 370.

[7] Kelley, E. F., Hebner, R. E., Fitzpatrick, G. J., Forester, E. O., "The Effect of Aromatic Impurities on the Positive Streamer Growth in Marcol 70," Conference Record of the 1984 IEEE International Symposium on Electrical Insulation, IEEE Publication 84CH1964-6, pp. $284-287$.

[8] Hebner, R. E., Kelley, E. F., Forester, E. O., Fitzpatrick, G. J., “Observation of Pre-breakdown and Breakdown Phenomena in Liquid Hydrocarbons, II. Non-uniform Field Conditions," IEEE Trans. EI, Vol. 20, 1985, pp 281-292.

[9] Leasint, O. and Jung, M., "Streamer Propagation in Cyclohexane: Influence of a Polyaromatic Additive,” Annual Report 1997 Conference on Electrical
Insulation and Dielectric Phenomena, IEEE Publication 97CH36046, pp. $672-675$.

[10] McKenny, P.J., and McGrath, P.B., "Anomolous Positive Point Pre-breakdown Behavior in Dielectric Liquids,” IEEE Trans EI, Vol 19, 1984, pp. $93-100$.

[11] Onsager, L., "Initial Recombination of Ions,” Phys. Rev., Vol. 54, Oct. 1938, pp. 554-557.

[12] Houser, N. and Jarnagin, R. C., "Electron Ejection from Triplet State in Fluid Solutions," J. Chem. Phys., Vol. 52, No. 3, Feb. 1970, pp.1069-1078.

[13] Venkatesh, S. K. and Naidu, M. S., "Experimental and Theoretical Investigations on the Statistics of Time Lags to Corona Inception and Breakdown of $\mathrm{SF}_{6}$ in Non-Uniform Electric Fields," Gaseous Dielectrics VIII, Christophorou, L. and Olthoff, J. eds., Plenum Press, New York, 1998, pp. $155-160$.

[14] Billar, P., "A Simple Qualitative Model for the Different Types of Streamers in Dielectric Liquids," Conf. Record 1996 International Conf. Conduction and Breakdown in Dielectric Liquids, July 1996, 189-192.

[15] Dacre, B. and Hetherington, J. I., "The Effects of Contaminants on the Behavior of Conductivity Improvers in Hydrocarbons," J. Electrostatics, Vol. 45, 1998, pp. 53-68. 